\title{
An Augmented Reality Platform inside PURESAFE project
}

\author{
Héctor Martínez and Seppo Laukkanen \\ SenseTrix \\ Helsinki, Finland \\ E-mail: \{hector.martinez, Seppo.laukkanen\}@sensetrix.com
}

\begin{abstract}
This paper presents the results of a three-year research project inside PURESAFE project. PURESAFE contains 15 research projects and aims to enhance the life-cycle management of facilities emitting ionizing radiation. The research project presented in this paper focuses in Augmented Reality solutions for maintenance purposes. The outcome of the research project is a new platform containing an Augmented Reality engine and an authoring tool for the creation of Augmented Reality applications for maintenance in large scientific facilities emitting ionizing radiation. The platform has been developed and tested in real conditions.
\end{abstract}

Keywords-augmented reality; maintenance; radioactive facilities; human intervention; remote handling

\section{INTRODUCTION}

PURESAFE [1] is an Initial Training Network (ITN) for the training of young researchers, funded under the European Commission's Seventh Framework Programme Marie Curie Actions. The aim of ITN projects is to provide new scientific knowledge and, at the same time, train new researchers targeting to complete doctoral studies.

PURESAFE (Preventing hUman intervention for incrREased SAfety in inFrastructures Emitting ionizing radiation) comprises 15 interdisciplinary research packages that aim to train 15 Early Stage Researchers (ESR) and to develop new means of cost-efficient life-cycle management of facilities generating ionizing radiation. The participants of the PURESAFE consortium are universities (Tampere University of Technology, Universidad Politécnica de Madrid and Karlsruhe Institute of Technology), international research organizations (European Organisation for Nuclear Research CERN- and Helmholtz Centre for Heavy Ion Research/Facility for Ion and Antiproton Research -GSI/FAIR) and industrial partners (SenseTrix, Oxford Technologies and bgator).

Every research package is focused on a specific aspect of the life-cycle management of facilities. From these packages, research package 11 (RP11) is titled "Augmented reality-based maintenance tool for hazardous places". The focus of RP11 is to design and develop AR-based tools to aid maintenance workers while performing maintenance tasks.

This paper presents an overview of the research work performed within PURESAFE RP11. The work has been carried out from July 2011 to June 2014. The rest of the text is structured as follows: section II describes the background of the project. In section III, a description of the work developed in the project is presented. The results of the research work are explained in section IV. Finally, the conclusions of the project are presented in section $\mathrm{V}$.

\section{BACKGROUND}

As mentioned in the introduction, PURESAFE is targeted to infrastructures emitting ionizing radiation. Therefore, human intervention and remote handling procedures may coexist in the maintenance systems of these facilities. Consequently, the work developed in RP11 has considered both types of maintenance.

The use of Augmented Reality (AR) for maintenance has already been considered in several studies (e.g. [2], [3], [4]). The typical approach followed in the majority of the cases is to use the technology to augment the devices to maintain with virtual instructions on the steps that have to be followed by the maintenance worker. The potential benefits are numerous, ranging from increasing the safety of the workers and minimising the accident risks to reduction of working times and maintenance costs.

Although the majority of the previous studies are targeted to human intervention maintenance, the benefits from the use of $\mathrm{AR}$ for remote handling maintenance have also been foreseen [5]. However, the studies presented in both maintenance types in the literature are prototypes targeted to solve a specific problem and they are usually not scalable and not flexible enough to be adapted to different maintenance procedures even within the same facility.

As a result of these considerations, scalable tools that combine both types of maintenance (human intervention and remote handling) are needed. Therefore, the approach followed by RP11 is to provide a new solution aiming to overcome this situation.

\section{AR PLATFORM}

The aim of RP11 is to develop several AR related techniques oriented to maintenance and to integrate those into a unique system that can be used in large scientific facilities. Maintenance in facilities emitting ionizing radiation is usually carried out in two ways: human intervention and remote handling. The purpose is to provide useful AR tools for both 
approaches. This means that some of the developments are human intervention oriented and some other techniques are remote handling oriented.

In both cases (human intervention and remote handling), the final application needs to be easy to use while helping the worker in the process of carrying out the work in a faster and safer way thus reducing the costs and risks of maintenance.

As a result of the previous considerations, a new platform integrating the proposed techniques and features has been developed. The platform comprises two main cores. On one side, a powerful AR engine has been developed. On the other side, an authoring tool for the creation of AR applications using the AR engine has also been developed.

The AR engine is mainly based on marker tracking, although it also includes a possibility for tracking images and point clouds. However, marker-based solution has been considered as the most suitable AR technique for the desired purpose, due to the restrictions and characteristics of the target facilities. Although the techniques for markerless AR have improved in the recent years, it is very complicated to provide a robust final solution that includes a large number of devices to be augmented in real time with the current software and hardware. On the other hand, marker-based AR provides a cost-efficient, robust and real-time solution for large scale projects, such as the case of scientific facilities where a large number of different devices have to be maintained.

Moreover, a new hybrid approach combining traditional AR markers with OCR (Optical Character Recognition) technology has been also developed and integrated into the platform. Fig. 1 shows an example of the design of the marker used in the hybrid approach. The goal of this approach is to provide a solution that allows the deployment of a coherent system in large scale projects.

Fig. 1. Example of the design of the marker included in the proposed hybrid approach. The marker is made up of a traditional $2 \mathrm{D}$ barcode marker and a text code. The 2D barcode marker is used for determining the position and orientation of the objects in the real world while the text code defines the content associated to each marker. The recognition of the marker is carried out by traditional AR techniques while the recognition of the text code is made using OCR.
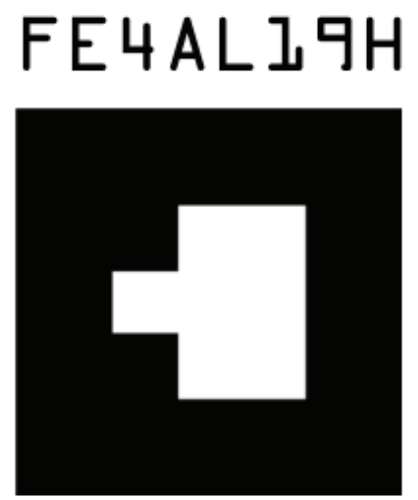

The authoring tool included in the platform allows nonprogrammers to develop AR applications targeted to maintenance procedures. The maintenance-oriented features included in the AR engine have been enabled in the authoring tool in order to easily allow users to utilise these features in the developed AR applications. The most relevant features included in the platform are the aforementioned hybrid approach, a step-by-step guiding system including keypoints for guiding remote maintenance and a maintenance-job-task structure of the applications. Fig. 2 shows a screenshot of the authoring tool.

Fig. 2. Screenshot of the implemented authoring tool. The screenshot shows a typical example of image definition to be used in the final augmented scene.

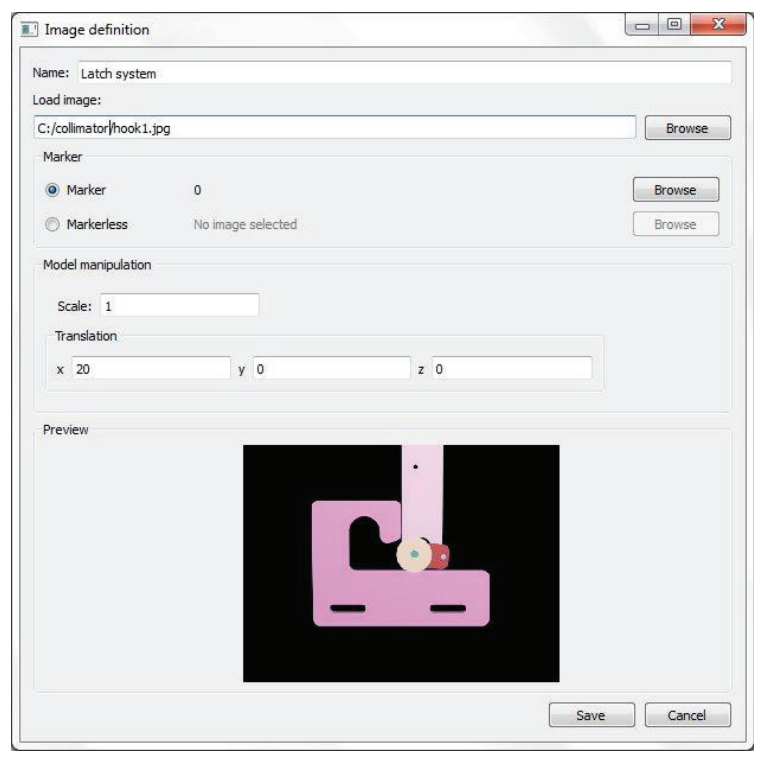

The platform provides facilities with a design that allows its deployment in large infrastructures. The platform makes use of the aforementioned hybrid approach combined with a database system in order to enable large deployments of AR applications within the same facility. The AR applications follow a maintenance-job-task structure that provides a coherent system for the creation, storage and use of the developed applications.

\section{RESULTS}

In this section, the results from this three-year work are presented. As explained in the previous section, the final outcome of the project is a new platform for AR solutions oriented to help in maintenance procedures in large scientific facilities, especially for those emitting ionizing radiation.

The platform includes a set of common AR features as well as new maintenance-oriented features. One important feature inside the platform is the design and implementation of a new hybrid approach (which combines traditional marker-based tracking with OCR technology to achieve more specific recognition) for the deployment of a coherent $\mathrm{AR}$ system in large facilities. The details of this hybrid approach can be found in [6]. 
The developed platform comprises an AR engine and an authoring tool. Both sides of the platform are meant to co-work closely and therefore the features from the engine can be used in the authoring tool for the creation of the AR applications. A comprehensive description of the developed platform can be found in [7].

The platform is targeted to human intervention maintenance and remote handling maintenance. Due to the important focus of PURESAFE project towards remote handling and the relatively low number of previous AR remote handling research studies, an important effort has been made in that direction. The developed features have been tested on a real environment where remote handling is required. The use case was the exchange of a collimator inside the Large Hadron Collider (LHC) at CERN. The prototype developed for the collimator exchange was tested on a real scale collimator inside a mock-up of the LHC tunnel. The results of the designed prototype have been detailed in [8]. Moreover, the publication also describes the difficulties related to remote handling maintenance in facilities emitting ionizing radiation, found during the development. To overcome these difficulties, specific solutions are also proposed. The aim is that the proposed solutions may be used as helping guidelines for future designs of similar systems.

Fig. 3 shows an image obtained from the tests carried out with the CERN use case. A crane is used to perform the collimator exchange. The use of the crane is done remotely and, therefore, the operator needs to rely on the visual information obtained by a camera. The information from the camera can be difficult to understand due to the lack of a third dimension. Therefore, additional information provided by the $A R$ view can enhance the understanding of the real situation.

In the image, the AR application provides the operator with a path to follow in order to proceed with the collimator exchange. The path is made up of keypoints that the operator has to follow to reach the final goal (also displayed with a virtual 3D model). When the procedure begins, only one keypoint is displayed at a time to clearly show which the current goal to reach is. The prototype has been tested in the facilities by CERN personnel and it has successfully worked in real time with a standard PC. For more details, please refer to [8].

Fig. 3. Image of the prototype developed for collimator exchange. In the image, a ste-by-step guiding system based on keypoints is displayed in order to aid the operator. When the exchange begins, one keypoint is displayed at a time, showing the goal to reach.

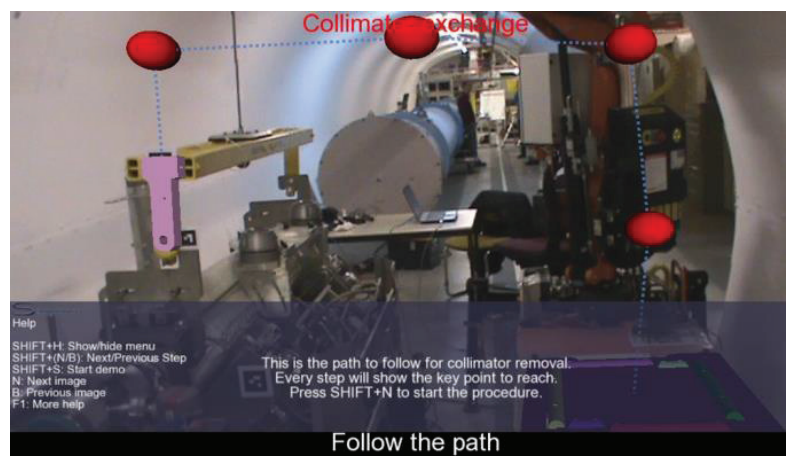

Finally, the platform has been developed to be flexible enough to be adapted to different situations and environments. This flexibility has already been demonstrated as the platform has been successfully utilised to develop a new AR-based educational and training solution, as explained in [9].

\section{CONCLUSIONS}

PURESAFE is an interdisciplinary project that involves academic and industrial partners. Therefore, the interactions between both partner types allow connecting research results with commercial solutions. The possibilities of interaction between partners also offer a wider view of the problems to be solved and therefore to implement an enhanced solution.

The 15 research packages included in PURESAFE comprise a wide variety of fields, including mechanical engineering, software engineering, robotics, management and innovation models and radiation protection. The final goal is to obtain better solutions for the management of facilities generating ionizing radiation.

One of these 15 research projects is targeted to provide new AR solutions for maintenance in the mentioned facilities. As it has been explained in this paper, the final outcome of the research project is a new platform that comprises an AR engine and an authoring tool for the easy creation of AR applications for maintenance.

At the end of the three-year project, the platform has been developed and tested in real facilities and it is ready for deployment in a real environment. In the post-project period, the platform will be enhanced by including new foreseen features and marketed to potential users in scientific facilities, especially in those emitting ionizing radiation.

\section{ACKNOWLEDGMENT}

This research project has been supported by a Marie Curie Initial Training Network of the European Community's Seventh Framework Programme under contract number (PITNGA-2010-264336-PURESAFE).

\section{REFERENCES}

[1] PURESAFE, http://webhotel2.tut.fi/iha/puresafe/

[2] F. De Crescenzio, M. Fantini, F. Persiani, L. Di Stefano, P. Azzari, and S. Salti, "Augmented reality for aircraft maintenance training and operations support," Computer Graphics and Applications, IEEE, vol. 31, no. 1, pp. 96-101, 2011.

[3] S. J. Henderson and S. K. Feiner, "Augmented reality in the psychomotor phase of a procedural task," in Mixed and Augmented Reality (ISMAR), 10th IEEE International Symposium on, pp. 191-200, 2011.

[4] S. Benbelkacem, M. Belhocine, A. Bellarbi, N. Zenati-Henda, and M. Tadjine, "Augmented reality for photovoltaic pumping systems maintenance tasks," Renewable Energy, vol. 55, pp. 428-437, 2013.

[5] R. King and D. Hamilton. "Augmented virtualised reality applications and benefits in remote handling for fusion," Fusion Engineering and Design, vol. 84, no. 2, pp. 1055-1057, 2009.

[6] H. Martínez, S. Laukkanen and J. Mattila, "A new hybrid approach for augmented reality maintenance in scientific facilities," International Journal of Advanced Robotic Systems, 10:321, DOI: 10.5772/56845, 2013. 
[7] H. Martínez, S. Laukkanen and J. Mattila, 2014, "A new flexible augmented reality platform for development of maintenance and educational applications," International Journal of Virtual Worlds and Human Computer Interaction, vol. 2, no. 1, 2014.

[8] H. Martínez, T. Fabry, S. Laukkanen, J. Mattila and L. Tabourot, "Augmented Reality aiding Collimator Exchange at the LHC," NIMA
(Nuclear Instruments and Methods in Physics Research Section A: Accelerators, Spectrometers, Detectors and Associated Equipment). (In press).

[9] H. Martínez and S. Laukkanen, "STEDUS, a new educational platform for augmented reality applications," 4th Global Conference on Experiential Learning in Virtual Worlds, 2014. 